\title{
Mechanical scanning probe nanolithography: modeling and application
}

\author{
P.M. Lytvyn ${ }^{1}$, O.S. Lytvyn ${ }^{1}$, O.M. Dyachyns'ka ${ }^{1}$, K.P. Grytsenko ${ }^{1}$, S. Schrader ${ }^{2}$, I.V. Prokopenko ${ }^{1}$ \\ ${ }^{1}$ V. Lashkaryov Institute of Semiconductor Physics NAS of Ukraine, 41, prospekt Nauky, 03028 Kyiv, Ukraine \\ ${ }^{2}$ Institute of Photonics, Laser and Plasma Technology, University of Applied Sciences Wildau, F.-Engels-Str. 63, \\ 15745, Wildau, Germany
}

\begin{abstract}
The paper presents a study on modeling the mechanical interaction between the tip of a scanning atomic force microscope (AFM) and surfaces of various types, which makes it possible to optimize parameters and modes for mechanical AFM nanolithography. The practical assessment of mechanical nanoprobe lithography based on the method of a direct surface patterning was carried out during fabrication of functional elements for molecular electronics. Polymethine dye nanowires of a specified configuration and the cross-section $3 \times 20 \mathrm{~nm}$ have been successfully formed in a multilayer polytetrafluoroethylene/gold/silicon nanostructure.
\end{abstract}

Keywords: scanning probe microscope, nanolithography, nanostructures.

Manuscript received 18.07.12; revised version received 03.09.12; accepted for publication 17.10.12; published online 12.12.12.

\section{Introduction}

The dimensions of components for integrated circuits are mainly determined by lithographic processes, which are, in their turn, constantly developing in accordance with the increasing requirements of micro- and nanoelectronics. The minimum size currently achieved is in the order of several tens of nanometers. Over the period of 50 years, industrial lithography has reduced the size of the functional elements of structures from $1 \mathrm{~cm}$ (first transistors in 1958) to $\sim 22 \mathrm{~nm}$ (the elements of processors, 2012) [1]. According to forecasts, the traditional methods of production will reach the maximum of their capabilities at around $22 \mathrm{~nm}$, while technology in 2016 will be based on the functional elements having a size of about $11 \mathrm{~nm}$. There are two main approaches in the nanometer size range. The first one began with the microelectronic technology and was based on principles of optical, electron-beam, ion-beam, $\mathrm{X}$-ray, extreme ultraviolet lithography [2-5]. Reducing the wavelength of light used for exposure of photoresist as well as usage of $\mathrm{X}$-rays and electron beams provide the possibility to create patterns with feature sizes less than $100 \mathrm{~nm}$.
The second approach, nanotechnological, is based on using a controlled solid state nanoprobe and is known as scanning probe lithography (SPL) [6, 7]. SPL can be successfully applied as a separate operation in a technological cycle and, also, can directly create functional elements of structures in the prototypes of leading-edge devices (such as dielectric layers, nanocontacts, active junctions, etc.).

The most low-cost and relatively easy to implement methods are those of mechanical scanning probe lithography. The probe of an atomic force microscope operating in contact or tapping modes can be used for both mechanical modifications of a resist film with its subsequent transferring and etching through a formed mask, as well as for direct removal of material by scratching or coining. In this case, the probe is used as a high precision point contact tool to form trenches in a resist or metal film covering the substrate surface. Direct mechanical manipulation can be done with great accuracy, but it is not always possible to obtain trenches with edges of acceptable quality because of their roughness. To achieve required characteristics of a pattern, it is necessary to optimize a number of parameters of surfaces as well as probes, and especially, 
the dynamic parameters of tip-surface interaction. When standard silicon or nitride probes are used, mechanical lithography can be applied on relatively soft substrates, and there occurs only local modification of the substrate surface topography without its qualitative transformation. Electrophysical properties under this technique do not change. This particular method of SPL is the focus of this paper.

\section{Theory and modeling of the tip - surface interaction}

Hertzian analytical solution of the problem for elastically deformed state of material that occurs when a ball is pressed into a plane $[8,9]$ is the foundation for the basic models of a contact interaction between the probe tip of a scanning probe microscope (SPM) and a surface. In a general case, the relationship between the interaction force and penetration depth of an n-sided regular pyramid with the angle of inclination $\theta$ and the apex in the form of a hemisphere with a radius $R_{c}$, which is not deformed by a contact with the surface, is defined as [10]:

$$
F(\delta)=\int_{\mathrm{A}} p^{*}(r, \varphi) \frac{f(r, \varphi)}{\delta *} r d r d \varphi
$$

where $\delta^{*}$ is the penetration depth of an ideal tip; $f(r, \varphi)$ is a function of the tip penetration; $p^{*}(r, \varphi)$ is an analytical function of the tip shape.

Let $a$ be the radius of the contact area, $b$ is radial distance to the transition point from the spherical tip to the edge of the pyramid (Fig. 1a). Assuming that $a<b$, $f(r)$ is a function of the shape of a spherical tip with radius $R_{c}$, and the Hertzian model for this case can be expressed as:

$$
F=\frac{4 E}{3\left(1-v^{2}\right)} R_{c}^{1 / 2} \delta^{3 / 2}
$$

where $E$ and $v$ are Young's modulus and Poisson's ratio for the tip, respectively.

If $a>b$, for a typical SPM probe in the form of a quadrangular pyramid, we have:

$$
\begin{array}{r}
F=\frac{2 E}{1-v^{2}}\left[\delta a-\frac{2^{1 / 2}}{\pi} \frac{a^{2}}{\tan \theta}\left(\frac{\pi}{2}-\arcsin \frac{b}{a}\right)-\frac{a^{3}}{3 R_{c}}+\right. \\
\left.+\left(a^{2}-b^{2}\right)^{1 / 2}\left(\frac{2^{1 / 2}}{\pi} \frac{b}{\tan \theta}+\frac{a^{2}-b^{2}}{3 R_{c}}\right)\right], \\
\delta=\frac{a}{\tan \theta} \frac{2^{3 / 2}}{\pi}\left(\frac{\pi}{2}-\arcsin \frac{b}{a}\right)-\frac{a}{R_{c}}\left[\left(a^{2}-b^{2}\right)^{1 / 2}-a\right] .
\end{array}
$$

If $b \rightarrow 0$, then we have a regular quadrangular pyramid, and the expression is presented as:

$$
F=\frac{1}{2^{1 / 2}} \frac{E \tan \theta}{1-v^{2}} \delta^{2} .
$$

Meanwhile, the effective radius of the contact is defined as $a=\delta \tan \theta / 2^{1 / 2}$.

In SPM, load force is determined by the value of elastic deformation of the cantilever tip:

$F=-k x, \quad k=\frac{E t^{3} w}{4 l^{2}}$.

Here, $E$ is Young's modulus for the cantilever; $t, w$, $l$ are thickness, width and length of the cantilever, respectively. At the same time, deformation is gauged by the SPM laser-optical measuring system and calculated using the calibration data of vertical displacement $(Z)$ and sensitivity of the measuring system. The example of SPM "load force - penetration depth" curves is presented in Fig. 1b.

Depending on the selected cantilever stiffness, it is possible to get the same displacement when applying different load forces $F$ (Fig. 2) and thus to have control over resolution through force:

$Z=\frac{F}{k}+\frac{a^{2}}{R_{c}} ; a=\left(\frac{3 F R_{c}\left(1-v^{2)}\right.}{4 E}\right)^{1 / 3}$.

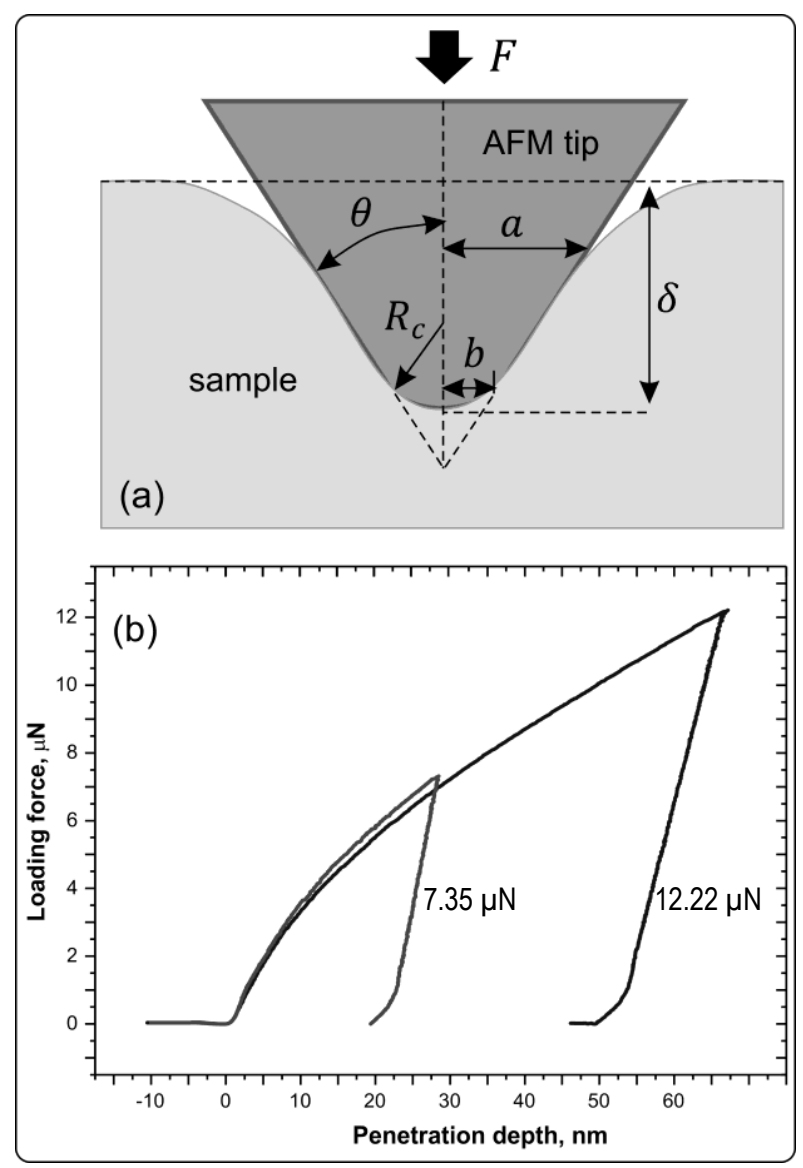

Fig. 1. Model of the hard tip penetration in a soft elastic surface (a); the experimental load - penetration curves of the quadrangular pyramidal silicon tip in a gold film (b) measured at different maximal loading forces [11]. 


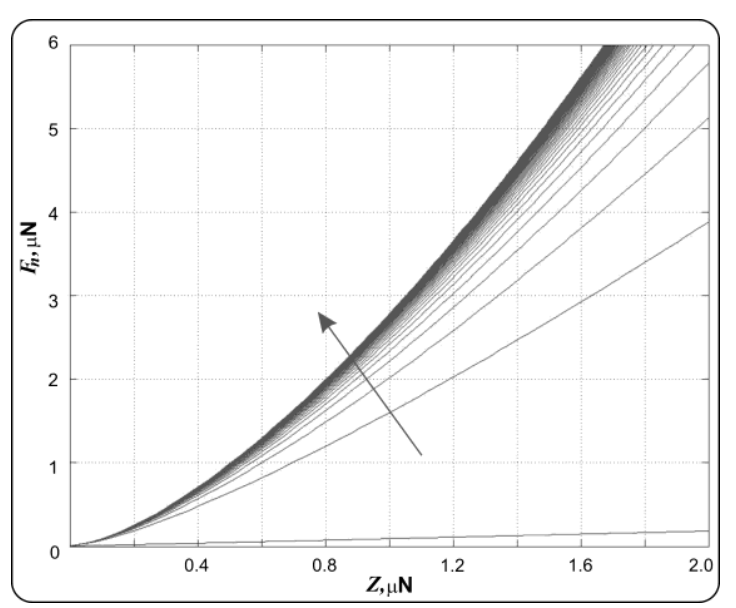

Fig. 2. The load force versus the vertical displacements of SPM probes with different stiffness. Stiffness increases in the direction of the arrow from 0.01 to $40 \mathrm{~N} / \mathrm{m}$ (increment of $0.5 \mathrm{~N} / \mathrm{m})$.

When a line (trench) is created by means of scratching the surface of material with a given force, its geometrical parameters can be described by a simple phenomenological relation:

$$
\begin{aligned}
& p\left(F_{n}\right)=\alpha_{1} \ln \left(F_{n} / F_{t 1}\right), \\
& m\left(F_{n}\right)=\alpha_{2} \ln \left(F_{n} / F_{t 2}\right),
\end{aligned}
$$

where $p$ is the depth and $m$ is the width of a line; $\alpha_{1}$ and $\alpha_{2}$ are coefficients that characterize the wedging and width of penetration, respectively; $F_{t 1,2}$ are corresponding threshold forces, after exceeding which the line is formed.

The value of the threshold force can be estimated from the following ratio:

$$
F_{t} \approx \frac{1.388 R_{c}^{2} H^{3}}{E^{*}}
$$

where $H, E^{*}$ are hardness and reduced Young's modulus of the sample.

Application of thin and soft layers provides several advantages: in addition to increasing resolution, it also prolongs the probe lifetime by preventing premature wear or an uncontrolled modification of the form of the tip apex, which happens in the case of engraving on hard surfaces. On the other hand, under these conditions (small load forces, soft plastic surface) the contribution of surface forces to the probe - surface interaction increases, and for a successful pattering process they must be taken into consideration.

The most widely used models describing this type of probe - surface interactions are those of JohnsonKendall-Roberts (JKR [12]) and Deryagin-Muller-Topov (DMT $[13,14])$. The phenomenon of a surface capturing a probe when it is approaching the critical distance is most often caused by long range attractive forces such as van der Waals forces. Whereas, when a probe is brought into a contact with a surface, there occur short-range force interactions, the most significant of which when operating in air are capillary forces arising due to the condensation of moisture on the surface of the probe and the sample [15].

The applicability of JKR, DMT or transitional Maugis model [16], for which the first two approximations are extreme cases, can be easily verified with the criterion $\mu$ proposed by Muller [17]:

$\mu=\frac{32}{3 \pi}\left(\frac{8 \gamma^{2} R\left(1-v^{2}\right)}{\pi E^{2} Z_{0}^{3}}\right)^{1 / 3}$,

where $Z_{0}$ is a typical interatomic distance, $\gamma$ is the surface energy. Under the condition that $\mu \ll 1$, the approximation of DMT theory is valid, meanwhile for $\mu \gg 1$, JKR model becomes applicable. Fig. 3 illustrates the dependence of the parameter $\mu$ on the probe radius for different values of the surface energy. It is evident that only at low values of the surface energy and small tip radii $\mu$ can approach 1 (approximation of DMT theory).

Using the Lenard-Jones potential, characteristic magnitude of van der Waals interaction can be estimated from the following relation [18]:

$$
F=\frac{A_{\mathrm{H}} R}{6 Z_{0}^{2}}\left(\frac{Z_{0}^{2}}{\delta}-\frac{1}{30} \frac{Z_{0}^{8}}{\delta^{8}}\right),
$$

Where $A_{\mathrm{H}}$ is the Hamaker constant. Fig. 4 illustrates the van der Waals interaction force for SPM probes with different radius and for different values of the surface energy (the Hamaker constant [19]).

The derivative of force with respect to distance $\partial F / \partial \delta$ as a function of distance $\delta$ (Fig. 5a) allows us to evaluate whether there will occur sticking to the surface of the probe with a given console stiffness. At the same time, modeling of JKR interaction for a probe with a radius of $30 \mathrm{~nm}$ at different values of the surface energy is shown in Fig. $5 b$.

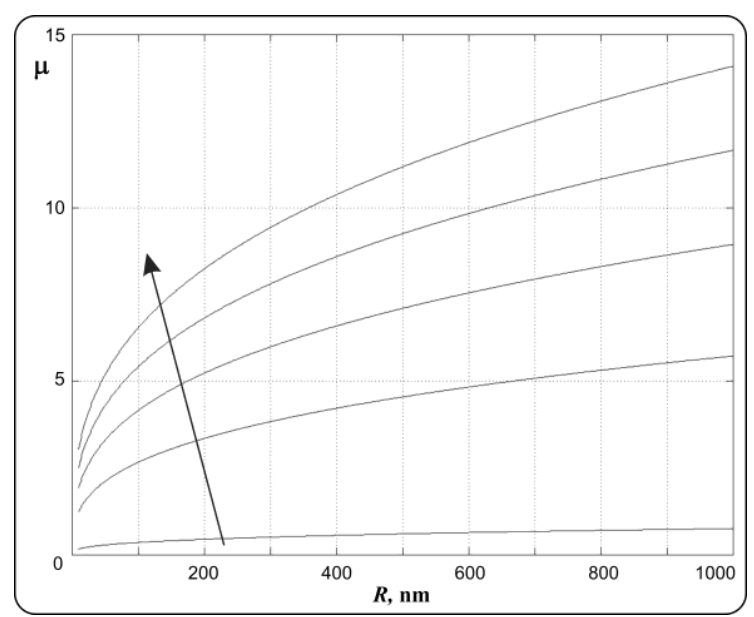

Fig. 3. Muller's parameter $\mu$ versus the tip radius of a SPM probe at the surface energy per unit area from 0.01 to $1 \mathrm{~N} / \mathrm{m}$ (increment of $0.02 \mathrm{~N} / \mathrm{m}$ ) as indicated by the arrow. 


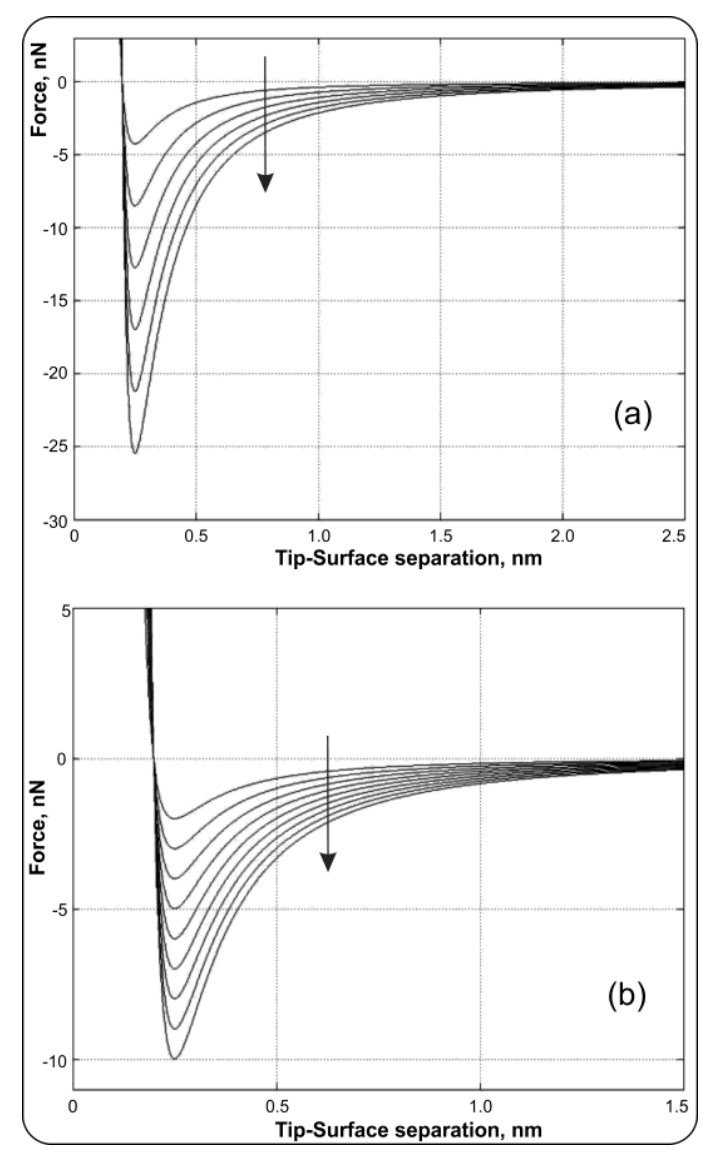

Fig. 4. The van der Waals interaction force versus the probe surface distance: (a) - for different values of the SPM probe radius $(5,10, \ldots 30 \mathrm{~nm})$; (b) - for different values of the Hamaker constant $(0.1,0.15, \ldots, 0.5 \mathrm{aJ}) . Z_{0}^{2}=0.35 \mathrm{~nm}$. Arrows indicate increasing of above mentioned parameters.

As it has been already noted, moisture condensation as well as the formation of water menisci and capillary bridges between the tip and surface leads to a significant increase in adhesive interactions observed in SPM. Within the simplest analytical models, it has been demonstrated that the maximum force of the capillary bridge rupture in the first approximation is calculated as $[20,21]$ :

$F(0) \approx 4 \pi \gamma_{L} \cdot R_{e f f}^{+} \cdot \varsigma$,

where $\gamma_{L}$ is surface tension for water $(72.8 \mathrm{mN} / \mathrm{m})$; $R_{\text {eff }}^{+}=\frac{R_{1} \cdot R_{c}}{R+R_{1}}$ is the effective curvature radius in the contact area between the sample and probe, here $R_{1}$ corresponds to the local curvature of the convex part of the sample surface, and $R_{c}$ is the curvature of the tip of the probe. For the area of the surface with negative curvature (depression, pit) $R_{\text {eff }}^{+}$is replaced by $R_{\text {eff }}^{-}$:

$$
R_{\text {eff }}^{-} \approx \frac{R \cdot R_{1}}{R_{1}-R_{c}} .
$$

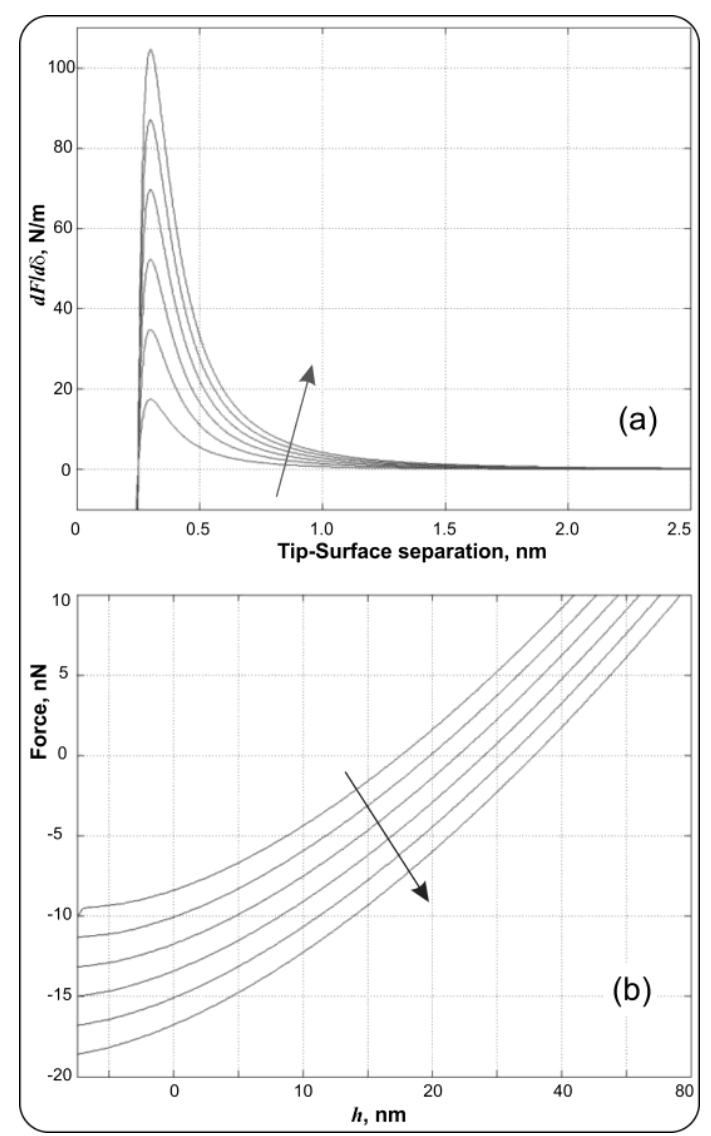

Fig. 5. Derivation $\partial F / \partial \delta$ versus probe - surface distance for the tip radii of $5,10, \ldots, 30 \mathrm{~nm}$ (a). JKR interaction forces for the tip radius of $30 \mathrm{~nm}$ at surface energies of $0.05,0.06, \ldots$ $0.10 \mathrm{~N} / \mathrm{m}$ (b). Arrows indicate increasing of above mentioned parameters.

Angles $\Theta$ and $\Phi$ are local wetting angles of the sample in the considered nanoarea and of the probe, respectively; and $\varsigma=(\cos \Theta+\cos \Phi) / 2$ is the average cosine of the wetting angle for two solid surfaces in contact.

According to the Young's formula [22], the macroscopic wetting angle is determined only by the surface energy of areas between which a capillary bridge is formed:

$\gamma_{L} \cos \Theta=\gamma_{S}-\gamma_{L S}$,

where $\gamma_{S}$ is the surface energy of solids, $\gamma_{L S}$ is the solid liquid interface energy.

It follows that capillary forces depending on the radius of the probe (from 30 to $50 \mathrm{~nm}$ ) can reach values of 20 up to $50 \mathrm{nN}$ for flat surface areas. For concave sections, however, the forces may exceed this value by $1.5-2$ times or more. This is one of the important factors that affect the quality and controllability of nanolithographical processes. In particular, the pressing force of the probe to the surface changes, which in its turn influences the friction forces that must be overcome for a horizontal contact motion. On a rough surface, the 
value of this additional force can vary considerably, which is especially relevant for softer materials like resists. Besides, this also affects the smoothness of manipulation with the probe on the stages of its penetration and withdrawing from the material, crossing existing grooves, etc. Furthermore, in the presence of surface phase inhomogeneity that is manifested in an inhomogeneous wetting angle, there arise some additional (determined by the gradient of the surface energy) forces acting along the surface, hindering or helping the horizontal movement of the probe.

\section{Results and discussion}

The practical implementation of mechanical lithography was carried out by means of NanoScope IIIa Dimension 3000 (Digital Instruments/Bruker AXS) SPM using specialized hardware oriented to the macros language NanoScript ${ }^{\mathrm{TM}}$ and commercial software code compiler $\mathrm{C}^{++}$. Test patterns were written on nanometer $(100 \mathrm{~nm})$ layers of gold, polytetrafluoroethylene (PTFE) and multilayer structures based on them (such as PTFE $(10 \mathrm{~nm}) / \mathrm{Au}(30 \mathrm{~nm}))$ formed on silicon substrates. Silicon, silicon nitride and diamond probes with tips of different configurations were used to perform the pattering. The shape of the probe tip was examined before and after manipulations applying the method described by us in [23]. As for the structures, they were manufactured by the technique of thermal deposition in vacuum. The accuracy with which layers of given thickness were formed was achieved through precise calibration of a quartz-crystal resonator in the deposition chamber via ex situ AFM measurements of the test layers thickness.

Fig. 6 and 7 demonstrate the selection result for the value of the load force necessary to form lines (trenches) with a specified depth in a film of gold on silicon. A series of lines were created with increasing loads and the obtained values of depth were compared with the calculated ones. It can be seen (Fig. 7) that the data correlate well with the only exception of the cases of significant depths where an effect the substrate produced on mechanical properties of the film becomes noticeable. As for uniformity of depth along the lines, the obtained values of deviations are approximately equal to the relief amplitude of a gold film. This indicates the stability of probe-surface force interaction, despite protrusions of accumulated material (pile-up) along the edges of the lines.

The problem of the pile-ups can be solved in two ways: first, by selection of the tip shape and choosing the optimal scanning direction (Fig. 8), and, second, by using thin resist layers. As can be seen, the patterning using a diamond tip in the shape of cube corner with the apex radius of about $30 \mathrm{~nm}$ in the direction along the probe cantilever creates symmetrical pile-ups (Fig. 8a). However, the patterning in the direction perpendicular to the cantilever produces pile-ups only on one side of the lines (Fig. 8b). Meanwhile the use of a silicon nitride probe with the same tip radius, but with the angle at the apex of $30^{\circ}$ ensures the formation of a clear line with minimal pile-ups (Fig. 8c).

Fig. 9 shows an example of test structures patterned on a PTFE $(30 \mathrm{~nm}) / \mathrm{Au}(80 \mathrm{~nm})$ film on silicon by means of a probe with the tip radius $10 \mathrm{~nm}$ and the angle at the apex close to $17^{\circ}$. It can be seen that at the intersection points the trajectory of the probe movement remains stable and a straight line is created. Such approach enables one to manufacture nanostructures by means of a technique known as "bottom-up". In this case, the shallow trenches are formed in the thickness of the insulator, a layer of PTFE, while the deep trench cut through to open the gold layer. Filling trenches with polymethine dye allowed us to obtain predefined nanowires of dye with the width of 20-100 nm and height ranged within 1 to $10 \mathrm{~nm}$. The shapes of wires could be controlled by the cross-section shape of nanopattern and dye deposition parameters (Fig. 10).

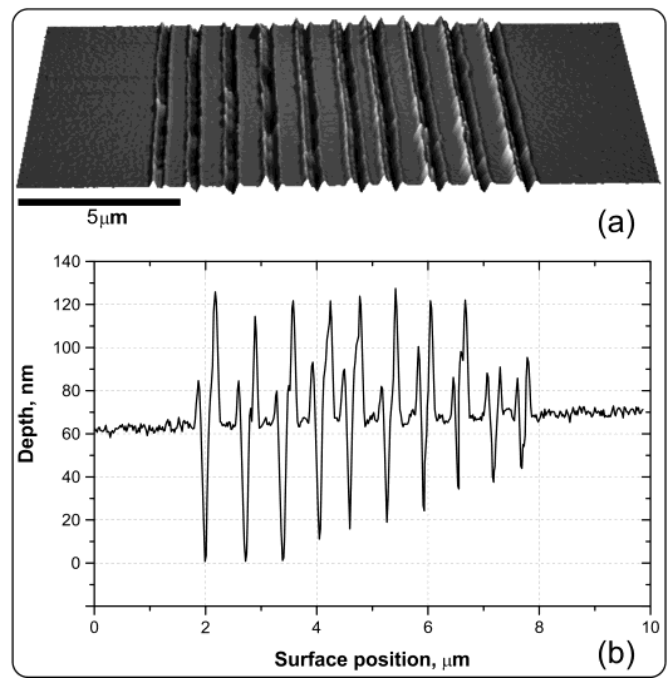

Fig. 6. A series of trenches in a gold film with a thickness of $80 \mathrm{~nm}$ created by means of mechanical lithography using a silicon probe at an increasing load force from 2.3 to $23 \mu \mathrm{N}$ in increment of $2.3 \mu \mathrm{N}$. AFM images of the surface (a); surface profile taken perpendicular to the lines (b).

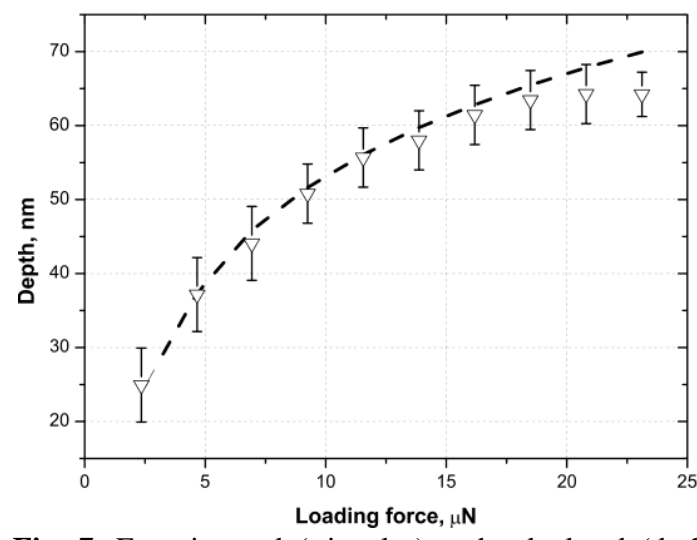

Fig. 7. Experimental (triangles) and calculated (dashed line) dependences of the trench depth on the loading force. 


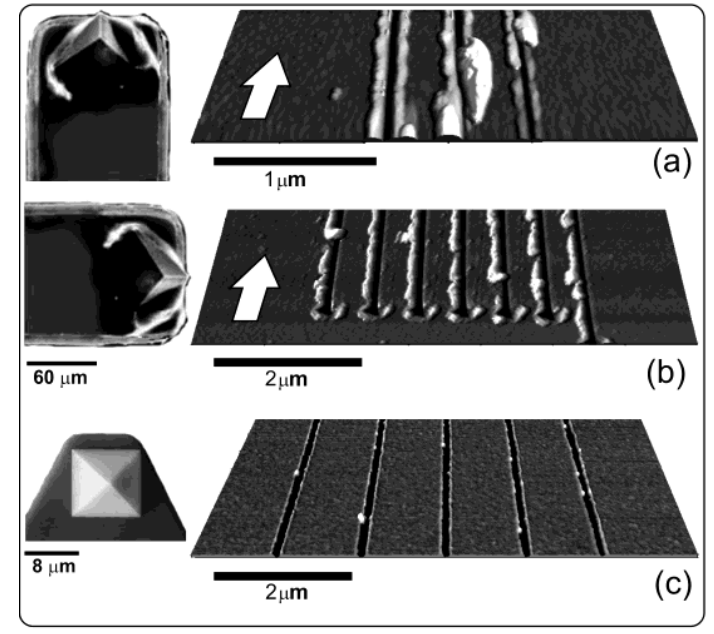

Fig. 8. Formation of pile-ups of different types on the edges of trenches depending on the motion direction of AFM tip as well as on its shape and size: diamond tip on a metal cantilever (the motion is perpendicular to (a) and along (b) the rear edge of the triangular pyramid of the probe); silicon probe with the tip in the shape of a regular quadrangular pyramid (c). The surface is a gold film on silicon.

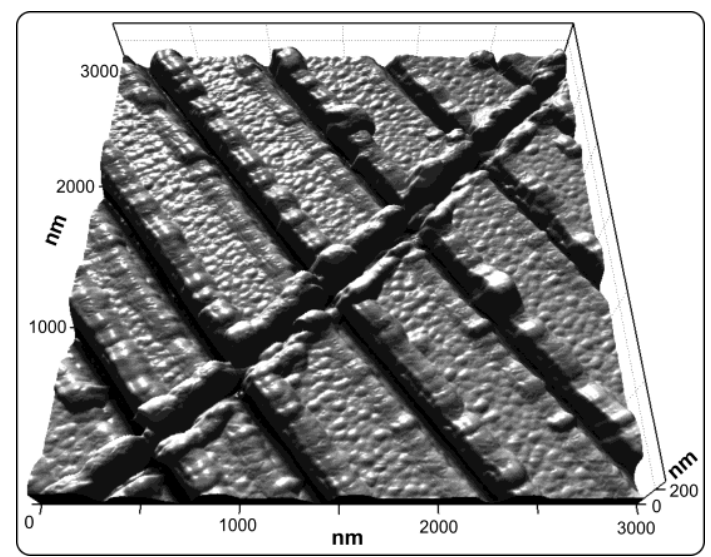

Fig. 9. 3D AFM image of series of perpendicular lines created on the PTFE/Au/Si structure by mechanical SPL technique.

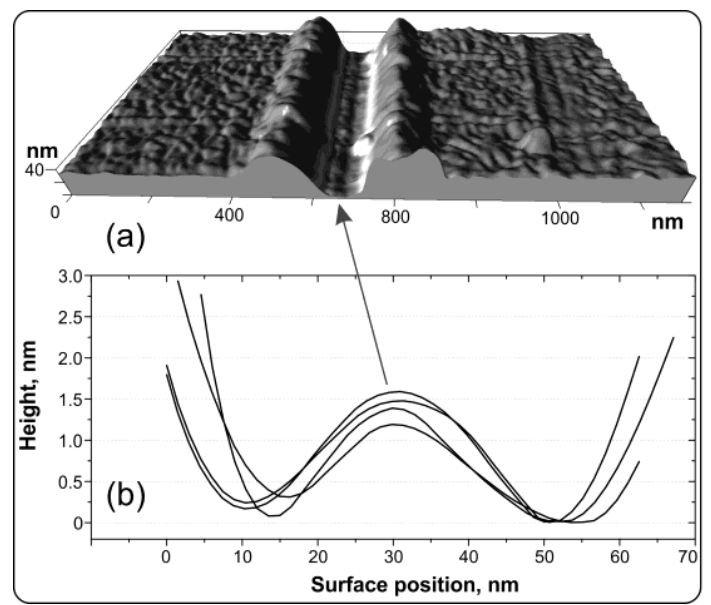

Fig. 10. Nanowire of polymethine dye localized in the trench of the PTFE layer cut through the underlying gold surface (a). Cross-sections taken in four various points along the nanowire (b).

\section{Conclusions}

In our study, we carried out a multilevel theoretical modeling of the interaction between the tip of an atomic force microscope and surfaces of different nature, which allowed us to find the optimal parameters for pattern application depending on the mechanical properties of material. Implemented was a prototype of practical nanoprobe lithography by the technique of a direct surface patterning through its mechanical modification. The method was tested in the course of fabrication of functional elements for molecular electronics. In particular, in the multilayer polytetraftorethylen/gold/ silicon nanostructure we successfully formed nanowires of polymethine dye of the $1.5 \times 20 \mathrm{~nm}$ cross-section size in predefined positions. The created prototype provides the possibility of a cheap and express technique for manufacturing a wide range of elements for test nanostructures of various purposes.

\section{References}

1. Intel ${ }^{\circledR} 22 \mathrm{~nm}$ Technology. http://www.intel.com/ content/www/us/en/silicon-innovations/intel-22nmtechnology.html.

2. G.P. Wiederrecht (Ed.), Handbook of Nanofabrication. - Elsevier, Academic Press, 2010.

3. D.G. Bucknall (Ed.), Nanolithography and Patterning Techniques in Microelectronics. Woodhead Publishing Ltd., 2005.

4. Yeonho Choi, Soongweon Hong, Luke P. Lee, Shadow Overlap Ion-beam Lithography for Nanoarchitectures // Nano Letters. 9, pp. 37263731 (2009).

5. M.S.M. Saifullah, K.R.V. Subramanian, E. Tapley et al., Sub-10 nm Electron Beam Nanolithography Using Spin-Coat able $\mathrm{TiO}_{2}$ Resists // Nano Letters. 3, pp. 1587-1591 (2003).

6. A.A. Tseng (Ed.), Tip-Based Nanofabrication: Fundamentals and Applications. Springer, 2011.

7. W.R. Bowen, N. Hilal (Eds.), Atomic Force Microscopy in Process Engineering. An Introduction to AFM for Improved Processes and Products. Elsevier Ltd., 2009.

8. H. Hertz, On the constant of elastic solids // $J$. Reine Angew. Math. 92, pp. 156-171 (1881).

9. H. Hertz. On hardness // Verh. Ver. Beförderung Geverbe Fleisses. 61, pp. 410-416 (1882).

10. F. Rico, P. Roca-Cusachs, N. Gavara et al., Probing mechanical properties of living cells by atomic force microscopy with blunted pyramidal cantilever tips // Physical Review E. 72, P. 021914 (10 pages) (2005).

11. P.M. Lytvyn, O.C. Lytvyn, A.A. Korchovyy et al., Mechanical scanning probe lithography for production of various purpose nanostructures // Proc. V Ukrainian scientific conference on physics 
of semiconductor (USCPS-5), Uzhgorod, Ukraine, p. 335 (2011).

12. K.L. Johnson, Contact Mechanics. Cambridge University Press; Reprintedition, 1987.

13. B.V. Derjaguin, V.M. Muller, Yu.P. Toropov, Effect of contact deformations on the adhesion of particles // J. Colloid. Interface Sci. 53, pp. 314326 (1975).

14. B.V. Derjaguin, Y.I. Rabinovich, N.V. Churaev, Direct measurement of molecular forces // Nature. 272, pp. 313-318 (1978).

15. B. Cappella, G. Dietler, Force-distance curves by atomic force microscopy // Surface Science Reports. 34, pp. 1-104 (1999).

16. D.J. Maugis, Adhesion of spheres: The JKR-DMT transition using a dug dale model // J. Colloid. Interface Sci. 150 (1), pp. 243-269 (1992).

17. V.M. Muller, V.S.Yushchenko, B.V. Derjaguin. On the Influence of Molecular Forces on the Deformation of an Elastic Sphere and Its Sticking to a Rigid Plane // J. Colloid. Interface Sci. 77, pp. 91-101 (1980).
18. D. Sarid, Exploring Scanning Probe Microscopy with mathematica. Weinheim: WILEY-VCH Verlag GmbH\&Co. KGaA, 2007.

19. B. Lennart, Hamaker constants of inorganic materials // Advances in Colloid and Interface Science. 70, pp. 125-169 (1997).

20. H.J. Butt, M. Kappl, Normal capillary forses // Advances in Colloid and Interface Science. 146 (1-2), pp. 48-60 (2009).

21. A.A. Efremov, P.M. Lytvyn, A.O. Anishchenko et al., Nanoprobe spectroscopy of capillary forces and its application for a real surface diagnostics // Semiconductor Physics, Quantum Electronics \& Optoelectronics. 13 (2), pp. 111-124 (2010).

22. J.N. Israelachvili, Intermolecular and Surface Forces. CA San Diego: Academic Press, 1998.

23. O.C. Lytvyn, P.M. Lytvyn, I.V. Prokopenko et al., Peculiarities of the nanostructures topometry by means of atomic force microscopy // Nanosystemy, nanomaterialy, nanotehnologii. 6 (1), pp. 33-44 (2008), in Ukrainian. 